\title{
Ruthenium-based Photocatalysis in Templated Reactions
}

\author{
Jacques Saarbach $\S^{\star}$, Eric Lindberg, and Nicolas Winssinger ${ }^{\star}$ \\ §SCS-Metrohm Award for best oral presentation in Medicinal Chemistry \& Chemical Biology
}

\begin{abstract}
Templated reactions proceed by bringing reagents in close proximity through their interaction with a template thus raising their effective concentrations. Templated reactions empower chemists to perform reactions at low concentrations in complex environments. Herein, we discuss our work on templated reactions leveraged on ruthenium photocatalysis. Over the past five years, we have used this reaction to uncage reporter molecules and sense or image nucleic acids or proteins of interest. The ruthenium photocatalysis chemistry has proven to be extremely robust and compatible with complex biological environments.
\end{abstract}

Keywords: Fluorescent probes · Nucleic acid sensing · Protein imaging · Ruthenium photocatalysis · Templated chemistry

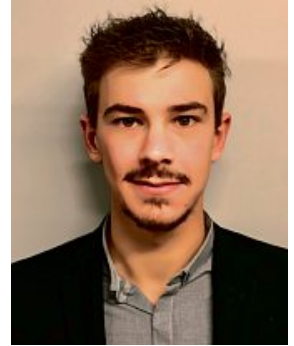

Jacques Saarbach was born and raised in the north east of France. He studied chemistry at the Ecole Nationale Supérieure de Chimie de Montpellier, with a focus on organic chemistry and biochemistry. In 2012, he moved to the USA in North Carolina to work on the synthesis of kinase inhibitors analogues in the laboratories of GSK. In 2013, he came back to Montpellier, to finish both a Master Degree in Chemistry and in Biology with a strong focus on medicinal chemistry. During this time, he worked on cell cycle biosensors and inhibitors at the IBMM developing peptide-based fluorescent probes for CDK cyclins. In 2014, he joined the group of Prof. Winssinger for a $\mathrm{PhD}$ at the University of Geneva, where his research focuses on the development of fluorescent probes based on templated reactions, mainly targeting protein kinases and on PNA-encoded chemical libraries screening for different proteins of interest.

${ }^{\star}$ Correspondence: J. Saarbach, Prof. Dr. N. Winssinger

Faculty of Science, Department of Organic Chemistry NCCR Chemical Biology, University of Geneva

30 quai Ernest Ansermet, $\mathrm{CH}-1211$ Geneva 4

E-mail: jacques.saarbach@unige.ch, nicholas.winssinger@unige.ch

\section{Introduction}

Selective organic chemistry transformations are usually achieved by careful choice of reagents and are typically carried out at relatively high concentrations (0.11 mol. $\left.\mathrm{L}^{-1}\right)$. In contrast, Nature manages selective transformations at lower substrate concentration $\left(10^{-2}-10^{-9}\right.$ mol. $\left.\mathrm{L}^{-1}\right)$ in complex mixtures. This is made possible by enzymes that increase the effective concentration of substrates through specific biosupramolecular interactions. Following the lead from Nature, chemists have designed templated reactions to increase the effective concentration of reagents and promote their reactions at dilutions where spontaneous reaction would not occur. The most frequently used templates for such chemical reactions are oligonucleotides where the design is facilitated by the programmability of Watson and Crick base pairing and tunable affinities. Templated reactions were first explored in the context of prebiotic chemistry, ${ }^{[1,2]}$ focusing on nucleic acid strand ligation. Pioneering work demonstrated that an oligonucleotide can template the ligation of complementary strands with activated phosphoester. Peptide nucleic acids (PNA) are DNA sur- rogates with a peptidic backbone based on repeating $N$-(2-aminoethyl)-glycine motifs (Fig. 1). ${ }^{[3]}$ PNAs hybridize according to the same Watson and Crick base paring rule as DNA or RNA but form more stable duplexes that are less sensitive to the ionic strength of the medium. Furthermore, the peptide chemistry involved in their synthesis is more malleable, facilitating modifications and conjugations. These features have made PNA an attractive platform to design assemblies with emerging function. ${ }^{[4]}$ Over the past decade, templated reactions and their applications have received significant attention, growing both in scope and breadth. ${ }^{[5,6]}$ Templated reactions have been used by Liu and coworkers to transform instructions encoded in nucleic acid into polymers or small molecules. ${ }^{[7,8]}$ Another important application of nucleic acid templated reactions has been for nucleic acid sensing. ${ }^{[9-11]}$ There is an important distinction between templated reactions leading to a ligation and a transformation without ligation, since in the latter case the reaction will turnover more readily, giving this strategy an advantage in the development of sensing and reporting systems with signal amplification (Fig. 2). This review will focus on recent

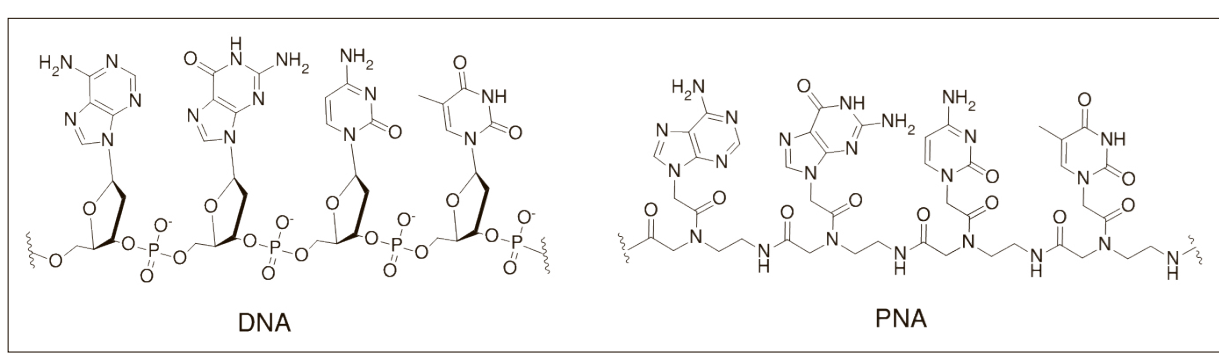

Fig. 1. DNA and PNA structures. 


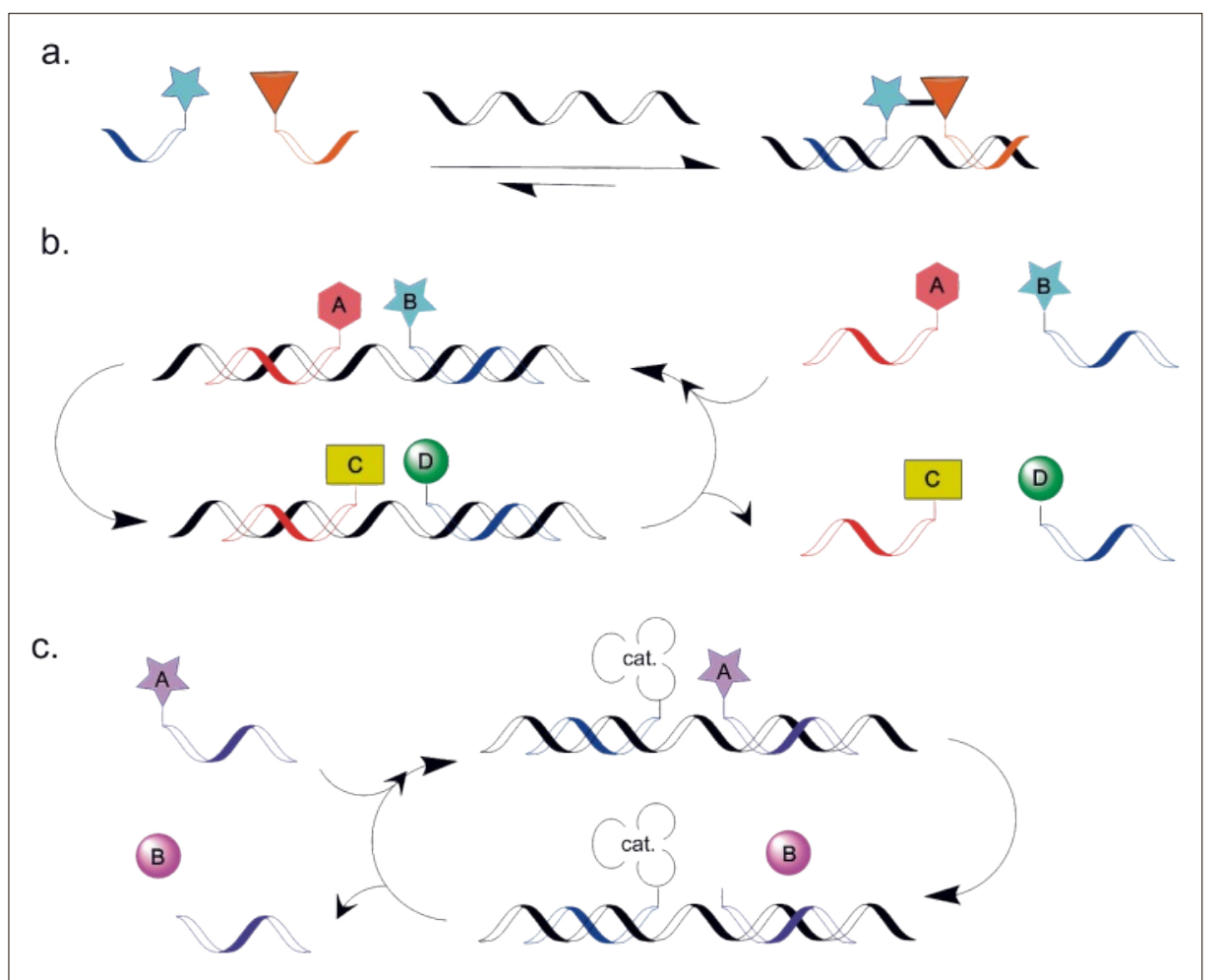

Fig. 2. Different types of templated reaction; a. templated strand ligation; b. templated reaction without ligation; c. catalytic templated reaction without ligation (one of the reagents is not consumed).

efforts using ruthenium photocatalysis in templated reactions.

\section{Ruthenium-catalyzed Templated Reactions}

\subsection{Azide Reduction}

We have been interested in templated reactions involving an azide reduction in light of the stability of the azide moiety and its bioorthogonality with cellular chemistry. We initially used the Staundinger reaction as a means of templated azide reduction. This reaction proved to be compatible with a wide range of profluorophores ${ }^{[12-14]}$ and was used to image RNA in live cells using GPNA (guanidine modified PNAs for improved cell permeability). ${ }^{[13,15]}$

Despite the success of this templated reaction in live cells, the Staundinger reaction suffers from background consumption of the phosphine reagent through oxidation. To overcome this limitation we investigated an alternative with ruthenium photocatalysis inspired by the demonstration that ruthenium tris-bipyridine can photocatalyze an azide reduction in presence of a stoichiometric amount of reducing agent (sodium ascorbate or NADPH). ${ }^{[16]}$ The reaction proved to work efficiently in a templated format, proceeding with only catalytic amount of the ruthenium photocatalyst-PNA conjugate. ${ }^{[17]}$ Since the ruthenium acts catalytically, this reagent does not need to dissociate from the temfertilization (left) or $36 \mathrm{~h} \mathrm{hpf} \mathrm{(right).}$ ting at $455 \mathrm{~nm}$. The fact that the reaction requires light adds an element of temporal control. ${ }^{[18]}$

Given the success of the reaction in cellulo, we turned our attention to more complex systems, namely zebrafish.[19] The absence of toxicity of the probes in zebrafish and the prolonged metabolic stability of PNAs raised the hope that the reaction could be used to sense miRNA in a living organism long after administration. To this end, we used PNA probes complementary to miRNAs of interest (Fig. 3a). After injection of the PNA probes in the embryo, the animals were raised in the dark for 24-36 h. The animals were irradiated for $30 \mathrm{~min}$ at $455 \mathrm{~nm}$ (1W LED lamp) and imaged to identify the localization of various miRNA of interest at either 24 or $36 \mathrm{~h}$ post fertilization (hpf) (Fig. 3b). For miR-9, at 24 hpf, a brain specific localization was observed and the brain to tail fluorescence ratio was much higher in the perfect match group than in the mismatch group, consistent with the known expression of this miRNA in the development of the zebrafish. The reaction still performed at $36 \mathrm{hpf}$, yielding a fluorescent signal that correlated to miRNA localization.

The increase in local concentration needed for templated reactions can also be achieved by the use of proteins and in particular, homodimeric proteins. Proteins have mostly been used in templated ligation reactions to create improved binders to the target. ${ }^{[20]}$ In the case of templated reactions without ligation, the signal amplification afforded by the reaction lends itself to the development of fluorescent probes that can report on protein dimerization. We used

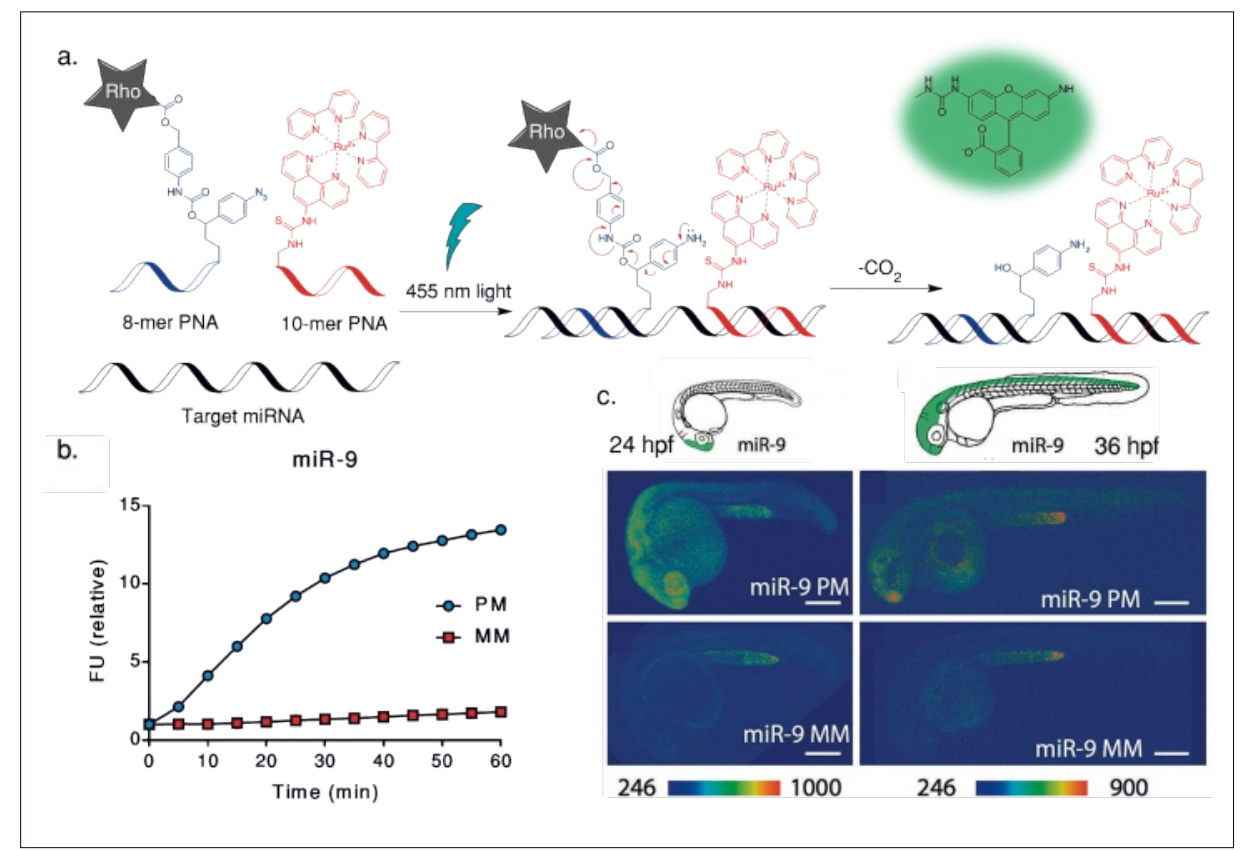

Fig. 3. a. Templated reaction used for in vivo imaging of miRNA. b. In vitro response of the miR-9 templated reaction. c. Theoretical localization and imaging of miR-9 in live zebrafish $24 \mathrm{~h}$ post 


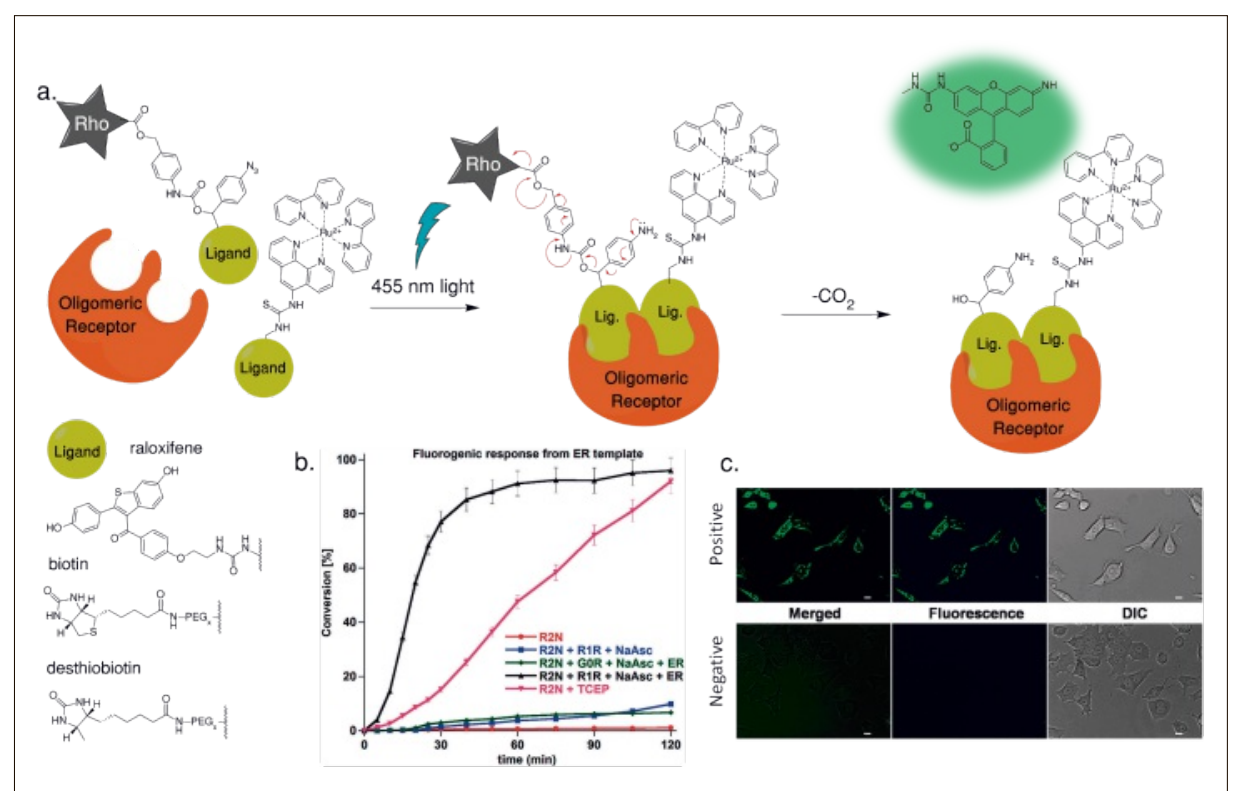

Fig. 4. a. Cartoon of protein homodimer templated reaction. b. In vitro fluorescence measurement of the reaction templated by the estrogen receptor dimer involving raloxifen ( $R X=$ raloxifen $+\mathrm{X}$ PEG spacers, $\mathrm{N}=$ rhodamine azide, $\mathrm{R}=$ ruthenium) probes with different linker lengths and a positive control (azide reduction by TCEP). c. Cellular imaging of estrogen receptor in MCF7 cells using raloxifen probes.

a pair of ligands derivatized with a caged rhodamine and a ruthenium photocatalyst, respectively (Fig. 4a). The reaction was shown to proceed with different oligomeric proteins (streptavidin or the estrogen receptor). In each case, the templated reaction proceeded at a rate greater than 30 -fold faster than the spontaneous reaction giving a clear difference in fluorescence after 30 min for template concentrations ranging from 0.1 to $1 \mu \mathrm{M}$. Interestingly, substrate turnover took place as in oligonucleotide templated reactions and linker length strongly impacted reaction rate underlining the importance of reagent alignment for optimal reactivity (Fig. 4b). Estrogen receptor dimerization could be monitored in live MCF7 cells using raloxifen as a ligand, derivatized with the two reactants (Fig. $4 c)$. The reaction performed just as well in pseudomonas aeruginosa, in which acetyl coenzyme A carbonylase could be targeted using the biotin probes, demonstrating the robustness of the reaction in cellulo.

\subsection{Pyridinium Reduction}

While the ruthenium-catalyzed azide reduction proved to be widely applicable in different settings, a faster reaction would be beneficial to accelerate detection and maximize turnover. To improve the kinetics of the reaction we turned to an N-methylpicolinium carbamate, which can be cleaved upon $\mathrm{Ru}(\mathrm{bpy})_{3}{ }^{2+}$ photoreduction. ${ }^{[21]}$ Using a model system involving two complementary PNA strands in stoichiometric amounts, the fastest templated reaction to date was achieved $\left(k_{a p p}=\right.$ $\left.0.138 \mathrm{~s}^{-1}, \mathrm{t}_{1 / 2}=5 \mathrm{~s}\right)$. However, the exceptional speed of this reaction made subconstants for different PNA lengths. beacon architecture which is opened in the presence of the target sequence, revealing a catalytic overhang. ${ }^{[22]}$

In order to accelerate the rate of product dissociation after templated reaction, we investigated a three-way junction architecture where the affinity of the product for the template-reagent complex is lower than that of the template-substrate complex. Three different systems were tested, including one system based on a triplex oligonucleotide PNA-PNA-DNA and one using a PNA-DNA in addition to a LysD-Ala-D-Ala vancomycin (KAA-Vanco) three-way junction architecture ${ }^{[23]}$ (Fig. 5b). Previous experiments showed that longer PNA probes increase the reaction rate $\left(k_{\text {app }}\right)$ but resulted in slower turnover in the catalytic reaction $\left(k_{\text {cat }}\right)$. The stoichiometric reaction rate $k_{\text {app }}$ of the three-way junction architecture involving vancomycin-D-Ala-D-Ala saturated at shorter PNA length than the control, thus establishing the contribution of vancomycin-ligand interaction to the supramolecular assembly. In the case of the catalytic reaction, the reaction rate $\left(k_{\text {cat }}\right)$ decreased beyond a 9-mer PNA due to product dissociation becoming the rate-limiting step. However, in the case of the three-way junction architecture, fresh substrate bearing the Lys-D-Ala-DAla interacted with vancomycin and in a process similar to oligonucleotide toehold exchange, displaced the PNA product due to higher affinity for the PNA-Ru(II)-DNA complex. In this case, a 6.4-fold improvement in turnover was observed using the three-way junction architecture.

Double-stranded RNA (dsRNA) has emerged as an important functional motif in cellular biology, motivating us to explore templated reactions that could dis-

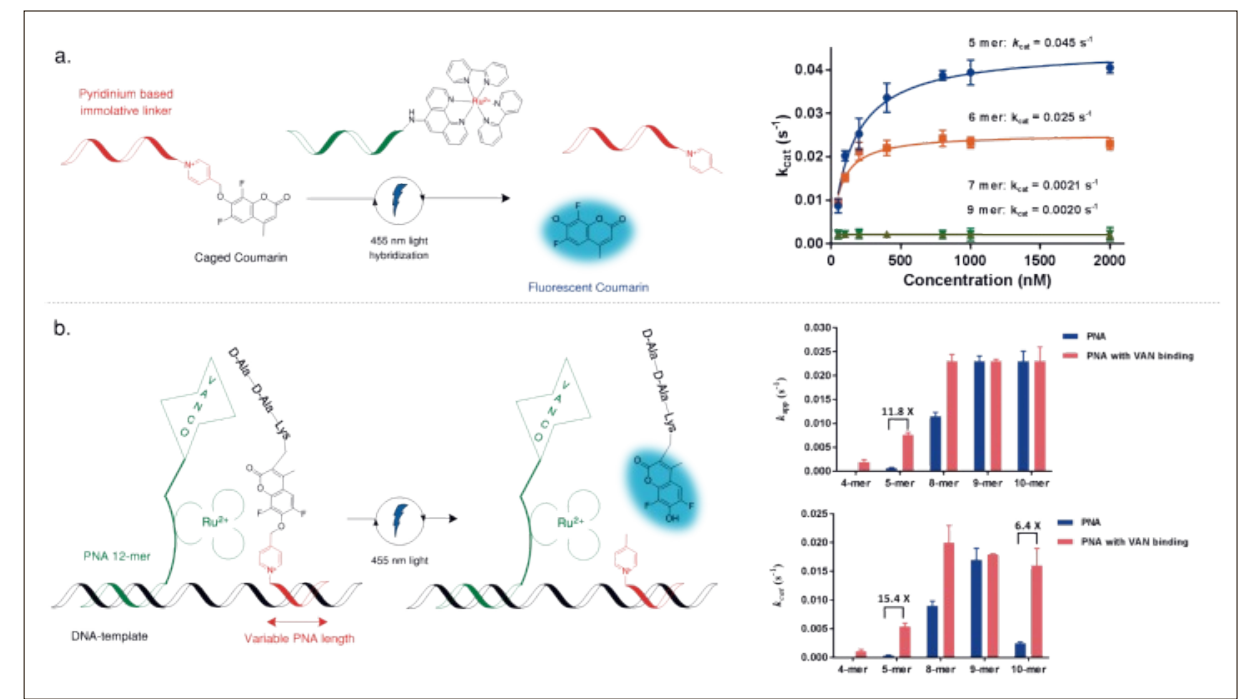

Fig. 5. a. PNA-templated ruthenium-catalyzed pyridinium reduction and kinetics of the reaction with various PNA lengths. b. Left: Structure of the three-way junction architecture: two PNA probes hybridized to their complementary DNA and D-Ala-D-Ala vancomycin complex bridging the two probes. Right: Values of kinetic constants for both stoichiometric kapp and catalytic kcat rate 
criminate between ssRNA and dsRNA. In this context, we took advantage of the progress made in the development of PNA monomers enhancing triplex formation of a PNA strand with dsRNA. ${ }^{[24,25]}$ By using two 9-mer PNA probes containing different amounts of $\mathrm{M}$ monomer to modulate total charge of the probe, we succeeded in developing a system that could hybridize to dsRNA in a triplex fashion and perform a templated reaction. Using only $20 \%$ of the ruthenium probe, the reaction reached about $80 \%$ conversion, again illustrating the benefit of the catalytic nature of the reaction. The reaction was shown to be specific for dsRNA; control reactions with ssRNA, ssDNA or dsDNA were comparable to the background reaction in the absence of template. A signal amplification corresponding to 19 turnovers (37\% conversion) was observed for $5 \mathrm{nM}$ of target dsRNA and 15 turnovers ( $3 \%$ conversion) were observed for dsRNA concentration as low as $0.5 \mathrm{nM}$ (Fig. 6). This reaction was found applicable to the detection of premiRNA 31; its abundance correlates to the function of the p53 pathway, an important tumor suppressor. ${ }^{[26]}$

\section{Kinase-templated Reactions}

Bi-substrate enzymes are another class of proteins suitable for templated chemical reaction. Among these, protein kinases stand out as an extremely attractive target class, with an ATP binding pocket and a substrate-binding site. These proteins are often involved in cancer, inflammatory diseases as well as neurodegenerative disorders and have been at the center of intense drug discovery efforts over the past two decades, providing a large panel of possible ligands. However, most of the inhibitors available to date target more than one kinase, making them unsuitable to discriminate the activity of a single kinase. We reasoned that the discrimination of kinases could be enhanced in templated reaction since it would require two reagents to bind simultaneously. While neither may bind exclusively to one kinase, the overlap of their selectivity should refine the overall discrimination to the kinase of interest. We decided to focus on dasatinib, ${ }^{[27]}$ a dual Src/Abl ATP-competitive inhibitor binding to the active conformation of the kinases. To create a catalytic cycle, dasatinib was modified with the previous ruthenium catalyst and an optimized peptide substrate for each kinase was modified with the caged rhodamine (Fig. 7a). ${ }^{[28]}$ Despite extensive work, we never obtained templated reaction with the azide-based self-immolative linker. This lack of reactivity was attributed to a dissociation rate that exceeds the reaction rate. To solve this issue, we ap-

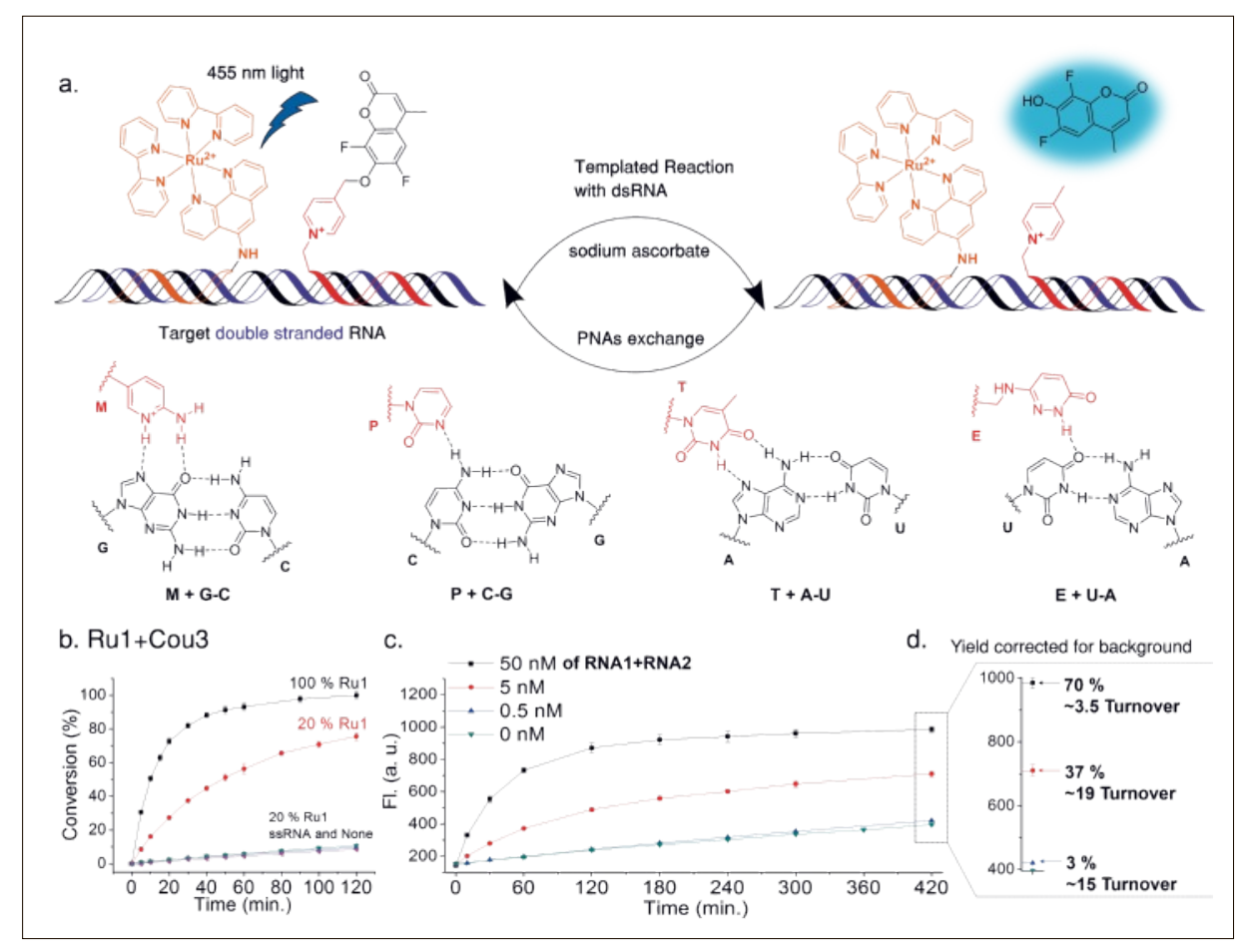

Fig. 6. a. dsRNA-PNA triplex templated reaction and PNA monomers used for triplex formation. b. Plot of the conversion for the templated reaction using ruthenium and coumarin PNA probes. c. Conversion of the templated reaction with different template dsRNA loading. d. Yield and number of turnovers of the reaction conditions used in $\mathrm{c}$.

pended a PNA dimer sequence before the caged rhodamine to increase the residency time of the peptide sufficiently for the reaction to proceed. This system displayed a 10-fold difference over background in twenty minutes and was able to work in fixed cells. In light of the faster reaction rate observed for the pyridinium reduction, we turned our attention to the pyridinium self-immolative linker and speculated that this reaction would proceed in the absence of the PNA adduct ( $k_{\text {off }}$ of the binders should be smaller than the $k_{\text {app }}$ of the reaction). Using this system, we managed to discriminate between Src and Abl both in vitro and in three different cell lines using ( $1 \mu \mathrm{M}$ of the rhodamine-peptide and $200 \mathrm{nM}$ of Dasatinib-Ru(II) probe), this reaction could also inform on the kinase activity of BCR-Abl/Abl in three different cancer cell lines depending on the expression of the oncogenic kinase BCR-Abl or simple WT Abl-1 (Fig. 7c).

\section{Conclusion}

The progress made in the development of templated reactions over the past decade has greatly expanded the toolbox available, broadening the field of applications for this technology. It has been shown that templated reactions can be used to respond to biological inputs in vitro, in cellulo and even in vivo. Thus, this technology is highly suitable for the development of biological probes for imaging or sensing of nucleic acids or proteins and could be applicable in the uncaging of prodrugs. The signal amplification obtained in these reactions can be maximized by reactions designed to yield products with lower affinity to the template (i.e. faster dissociation kinetics) than the substrate. The performance of some of these reactions are already comparable to the performance of enzymatic reactions. Ruthenium photocatalyzed reactions have proven very attractive for their biocompatibility and the temporal control that photochemical process affords. Amongst bioorthogonal reactions, few have been reported to be effective in live organisms putting ruthenium photocatalysis in a privileged reactivity space.

\section{Acknowledgements}

Jacques Saarbach is grateful to the Swiss Chemical Society and Metrohm for the best oral presentation in Medicinal Chemistry and Chemical Biology award. This work has been supported by the NCCR Chemical Biology and the Swiss National Science Foundation (SNF).

Received: January 29, 2018

[1] L. E. Orgel, Acc. Chem. Res. 1995, 28, 109.

[2] L. E. Orgel, Crit. Rev. Biochem. Mol. Biol. 2004, 39, 99 .

[3] M. Egholm, O. Buchardt, L. Christensen, C. Behrens, S. M. Freier, D. A. Driver, R. H. Berg, S. K. Kim, B. Norden, P. E. Nielsen, Nature 1993, 365, 566

[4] S. Barluenga, N. Winssinger, Acc. Chem. Res. 2015, 48,1319 . 


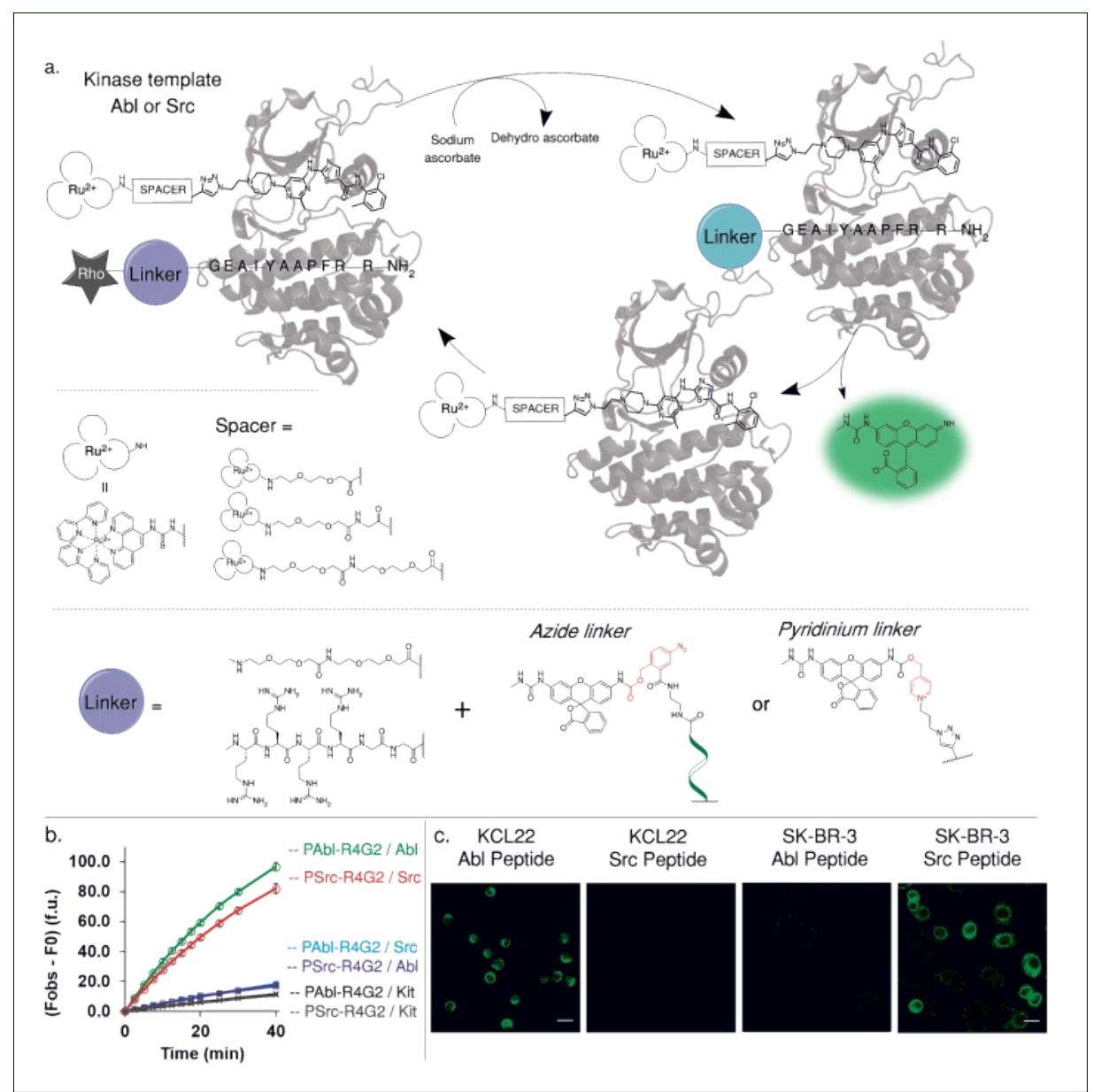

Fig. 7. a. Schematic representation of fluorophore release through $\mathrm{Ru}(I)$ catalyzed kinase templated reaction. b. Fluorescence output of the in vitro reaction using $1 \mu \mathrm{M}$ of the peptide-rhodamine probe and $100 \mathrm{nM}$ of dasatinib-Ru(II) and kinase template. c. Fluorescence imaging of the reaction in fixed cells with different peptide probes in KCL-22 and SK-BR-3 respectively Abl and Src driven cell lines.
[5] K. Gorska, N. Winssinger, Angew. Chem. Int. Ed. 2013, 52, 6820 .

[6] M. Di Pisa, O. Seitz, ChemMedChem 2017, 12, 872.

[7] X. Li, D. R. Liu, Angew. Chem. Int. Ed. 2004, $43,4848$.

[8] J. Niu, R. Hili, D. R. Liu, Nat. Chem. 2013, 5 , 282.

[9] A. P. Silverman, E. T. Kool, Chem. Rev. 2006 106, 3775.

[10] D. M. Kolpashchikov, Chem. Rev. 2010, 110 , 4709.

[11] A. Shibata, H. Abe, Y. Ito, Molecules 2012, 17, 2446.

[12] Z. L. Pianowski, N. Winssinger, Chem. Commun. 2007, 3820.

[13] Z. Pianowski, K. Gorska, L. Oswald, C. A. Merten, N. Winssinger, J. Am. Chem. Soc. 2009, 131, 6492.

[14] K. Gorska, A. Manicardi, S. Barluenga, N. Winssinger, Chem. Commun. 2011, 47, 4364.

[15] K. Gorska, I. Keklikoglou, U. Tschulena, N Winssinger, Chem. Sci. 2011, 2, 1969.

[16] Y. Chen, A. S. Kamlet, J. B. Steinman, D. R. Liu, Nat. Chem. 2011, 3, 146.

[17] M. Rothlingshofer, K. Gorska, N. Winssinger, Org. Lett. 2012, 14, 482.

[18] K. K. Sadhu, N. Winssinger, Chem. Eur. J. 2013, 19, 8182.

[19] L. Holtzer, I. Oleinich, M. Anzola, E. Lindberg, K. K. Sadhu, M. Gonzalez-Gaitan, N Winssinger, ACS. Cent. Sci. 2016, 2, 394.

[20] S. K. Mamidyala, M. G. Finn, Chem. Soc. Rev. 2010, 39, 1252.

[21] J. B. Borak, D. E. Falvey, J. Org. Chem. 2009 74, 3894.

[22] D. Chang, E. Lindberg, N. Winssinger, $J . A m$. Chem. Soc. 2017, 139, 1444.

[23] D. Chang, K. T. Kim, E. Lindberg, N. Winssinger, Bioconjug. Chem. 2018, 29, 158.

[24] T. Zengeya, P. Gupta, E. Rozners, Angew. Chem. Int. Ed. 2012, 51, 12593.

[25] T. Endoh, D. Hnedzko, E. Rozners, N Sugimoto, Angew. Chem. Int. Ed. 2016, 55, 899.

[26] K. T. Kim, D. Chang, N. Winssinger, Helv Chim. Acta 2018, doi: 10.1002/hlca.201700295.

[27] N. P. Shah, C. Tran, F. Y. Lee, P. Chen, D Norris, C. L. Sawyers, Science 2004, 305, 399.

[28] J. Saarbach, E. Lindberg, S. Folliet, S. Georgeon, O. Hantschel, N. Winssinger, Chem. Sci. 2017, 8, 5119. 